\title{
La radiobiologie française : historique, constats et enjeux
}

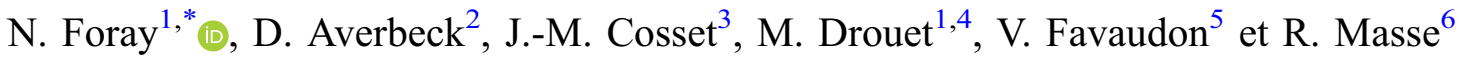 \\ ${ }^{1}$ Inserm UMR 1296, Centre Léon-Bérard, 28 Rue Laennec, 69008 Lyon, France. \\ ${ }^{2}$ Direction de la Recherche Expérimentale-D3P, CEA, 92260 Fontenay-aux-Roses, France. \\ 3 Centre de radiothérapie Charlebourg, Groupe Amethyst, 92250 La Garenne-Colombes, France. \\ ${ }^{4}$ Institut de Recherches Biomédicales des Armées (IRBA), 91220 Brétigny-sur-Orge, France. \\ ${ }^{5}$ Institut Curie, Inserm U 1021-CNRS UMR 3347, Université Paris-Saclay, Centre Universitaire, 91401 Orsay, France. \\ ${ }^{6}$ Centre Antoine Béclère, 45 rue des Saints-Pères, 75006 Paris, France.
}

Reçu le 28 septembre 2021 / Accepté le 12 octobre 2021

\begin{abstract}
Résumé - Forte des travaux pionniers de ses figures historiques emblématiques comme Pierre et Marie Curie, Claudius Regaud ou Antoine Lacassagne, la radiobiologie française se trouve aujourd'hui dans une situation dramatique en matière de ressources humaines et d'expertise, comme la plupart des pays développés. Pourtant, les progrès considérables dans la thérapie des cancers et dans le radiodiagnostic, les enjeux de Défense Nationale et de l'exploration spatiale ainsi qu'une attente sociétale grandissante pour une évaluation objective des risques liés aux radiations ionisantes devrait encourager nos institutions à maintenir et préserver le savoir et le savoir-faire des radiobiologistes. Sans proposer un catalogue des thèmes et des laboratoires, l'objectif de cet article, est de mieux analyser et comprendre l'évolution historique et scientifique de la radiobiologie française pour mieux en dégager les enjeux de demain.
\end{abstract}

Mots clés : radiobiologie / radiations ionisantes / politique de recherche

\begin{abstract}
Radiobiology in France: history, statements and issues. Despite of the pioneering works of its emblematic historical figures such as Pierre and Marie Curie, Claudius Regaud and Antoine Lacassagne, French radiobiology today finds itself in a dramatic situation in terms of human resources and expertise, like most developed countries. However, the considerable advances in cancer therapy and in radiodiagnosis, the issues of National Defense and space exploration as well as a growing societal expectation for an objective assessment of the risks linked to ionizing radiation should encourage the French institutions to maintain and preserve the knowledge and know-how of radiobiologists. Without offering a catalog of themes and laboratories, the objective of this article is to better analyze and understand the historical and scientific evolution of French radiobiology in order to better identify the challenges of tomorrow.
\end{abstract}

Keywords: radiobiology / ionizing radiations / research policies

\section{Introduction}

Dans son éditorial du numéro 3 de 2020 de Radioprotection, intitulé «Plaidoyer pour un soutien fort et durable à la recherche en radiobiologie pour optimiser la radioprotection», le rédacteur en chef indiquait "qu'il y a un besoin persistant de mise à jour des données de la radiobiologie. » ... «Nous ouvrons le journal Radioprotection à des articles destinés à encourager la communauté de la radiobiologie et au-delà les praticiens médicaux et les utilisateurs de radiations ionisantes à échanger idées et recherches pertinentes pour démontrer aux autorités compétentes la nécessité

\footnotetext{
$\overline{\text { *Auteur de correspondance }}$ : nicolas.foray@inserm.fr
}

d'investir davantage dans la recherche en radiobiologie et dans ses applications » (Bourguignon, 2020).

L'objectif de cet article, dans la droite ligne de cet appel, est de faire le point sur la radiobiologie française à travers son évolution historique et scientifique pour en tirer des leçons et voir plus clair dans la situation dramatique dans laquelle elle se trouve aujourd'hui, notamment en matière de ressources humaines et d'expertise.

\section{Contexte général}

Aujourd'hui, plus d'un siècle après la découverte des rayons $\mathrm{X}$ et de la radioactivité, de nombreux aspects de la réponse aux radiations ionisantes(RI) restent encore mal 
compris. Cependant, la dose moyenne d'exposition médicale annuelle ne cesse de croître. Parallèlement, l'attente sociétale pour une évaluation objective du risque lié aux RI et à leurs vecteurs est de plus en plus grande. Voici quelques éléments plus précis pour mieux comprendre le contexte actuel:

- en radiothérapie anti-cancéreuse : l'évolution technologique considérable notamment en termes de moyens balistiques ou d'association chimio-radiothérapie, pose des questions radiobiologiques nouvelles concernant les effets liés aux fractionnements de la dose (ex: radiothérapie hypo- ou hyper-fractionnée), au débit de dose (ex: FLASH-thérapie), à l'efficacité des particules lourdes (hadron-thérapie) et à la synergie entre radiations et agents biochimiques (ex: drogues, nanoparticules, anticorps,...) (Mazeron et al., 2005; Joubert et al., 2011; Cosset et al., 2013 ; Mazal et al., 2020; Friedl et al., 2021).

- en radiodiagnostic : au moins un quart de la population a un examen radiologique par an $(50 \%$ si on inclut les expositions dentaires) et la dose moyenne d'exposition médicale annuelle par patient dépasse $1 \mathrm{mSv}$, valeur de la limite de dose pour le grand public. L'Académie de Médecine a d'ailleurs alerté l'opinion sur le manque de justification de nombreuses expositions en radiodiagnostic. Les risques liés aux faibles doses de radiation constituent un des enjeux majeurs des recherches en radiobiologie: leur évaluation et leur gestion doivent faire l'objet de recommandations reposant sur des bases scientifiques solides (Médecine, 2015). Depuis février 2019, l'Autorité de Sûreté Nucléaire (ASN) a introduit la prise en compte de la radiosensibilité individuelle dans la justification des expositions diagnostiques (ASN, 2019).

- en génétique : environ 5 à $20 \%$ des individus sont radiosensibles : le développement récent de tests prédictifs de la radiosensibilité individuelle, d'abord issus d'entités académiques et bientôt proposés par des start-ups, pose des questions nouvelles d'ordre réglementaire, juridique et éthique qui doivent être résolues rapidement avant toute commercialisation. De plus, le lien entre radiosensibilité et certaines mutations génétiques reste encore méconnu (Foray et al., 2016).

- dans le cadre de l'exploration spatiale : l'exploration spatiale requiert une meilleure connaissance des effets biologiques des radiations cosmiques: ce domaine inclut les effets spécifiques aux particules lourdes, aux faibles doses et aux faibles débits de doses (Restier-Verlet et al., 2021)

- en environnement: avec l'identification des effets spécifiques imputables aux radionucléides dispersés dans l'environnement, la radiobiologie devient également environnementale avec des interactions nombreuses avec les géologues, les botanistes, les experts des différents écosystèmes biologiques. Par exemple, la remédiation des sols contaminés est désormais un sujet d'étude à part entière (Ding et al., 2016; Cannon et Kiang, 2020).

- en matière de sécurité : les effets liés aux rayonnements ionisants restent un sujet de préoccupation de sécurité nationale auxquels s'ajoute la résurgence du risque nucléaire et radiologique face à l'évolution du cycle de conflictualité qui fait réapparaître de nouveaux rapports de force en particulier au niveau étatique (Drouet et Ménager, 2019). La réponse biologique aux fortes doses de radiation, la radiopathologie, la dosimétrie biologique et le développement de contre-mesures médicales fondées sur l'avancée des connaissances fondamentales et adaptées à un traitement de masse sont au premier plan de la recherche duale civilo-militaire dans le domaine. Enfin, la pandémie due au Covid-19 nous a montré toutes les difficultés inhérentes à la gestion d'une crise majeure comme pourrait l'être un accident nucléaire sur notre territoire (Drouet et Herodin, 2014; Gagna et al., 2016; Riccobono et al., 2018; Drouet et Ménager, 2019; Cavallero et al., 2020; Endesfelder et al., 2021).

- en sciences humaines et sociales: la perception des risques liés aux radiations peut différer selon que l'on est scientifique, médecin ou patient, radiosensible ou radiorésistant, expert ou grand public ou que l'on évolue dans un cabinet ministériel ou dans une entreprise. L'étude psychosociale de ces différences pourrait aider dans la nature et la forme de l'information donnée par les « experts » aux «nonexperts ». Là encore, la pandémie liée au Covid-19 nous a montré combien peut être différente, entre individus, la perception du risque et comment peut être puissante l'influence des croyances. L'introduction des sciences humaines et sociales en radiobiologie apparaît donc aujourd'hui comme indispensable (Britel et al., 2015, 2018; Lindberg, 2021).

Les effectifs et les moyens structurels français en radiobiologie humaine peuvent-ils répondre à tous ces enjeux sociétaux, scientifiques et médicaux ? Répondre à cette question est l'objectif principal de cet article. Toutefois, nous n'avons pas souhaité réaliser un catalogue actualisé des laboratoires considérés comme « laboratoires de recherche en radiobiologie » ni des sujets les plus innovants. Au contraire, nous avons souhaité rappeler les différentes tendances successives, réussites ou échecs, «âges d'or» ou «périodes de disette» de la radiobiologie française pour mieux comprendre la situation aujourd'hui. Nous nous sommes délibérément basés sur des chiffres, ceux des thèses soutenues, des effectifs de recrutements et des articles publiés dans des revues internationales.

\section{La radiobiologie: définition}

La radiobiologie (encore appelée biologie des radiations) est l'étude des effets biologiques des RI. La radiobiologie concerne toutes les recherches sur les événements produits par les radiations ionisantes à toutes les échelles de temps et d'espace. C'est notamment le cas :

- des aspects physiques (ex: microdépôts d'énergie déposés dans la matière irradiée);

- des aspects chimiques (ex: espèces radicalaires et moléculaires issues notamment de la décomposition de l'eau irradiée);

- des aspects biomoléculaires (ex : production et réparation des dommages radioinduits de l'ADN et des chromosomes);

- des aspects cellulaires (ex: différents types de mort cellulaire radioinduite);

- des aspects tissulaires (ex: différents types de réactions notamment inflammatoires des tissus ou des organes irradiés);

- des aspects macroscopiques (ex: impacts sur les organismes, les populations et les écosystèmes) 
Par extension, la radiobiologie a un lien avec d'autres disciplines comme :

- la radioprotection, avec ses développements technologiques (dosimétrie) ou chimiques (contre-mesures) mais aussi en orientant ses principes et ses recommandations;

- la radiologie, la radiothérapie et la médecine nucléaire à travers la mise au point de thérapies innovantes et de diagnostics plus efficaces, de tests prédictifs des possibles séquelles post-irradiation (réactions tissulaires ou cancers radioinduits);

- l'anatomo-pathologie et notamment la cytogénétique, qui fut à la base des premiers tests pour identifier des maladies génétiques (notamment à travers les aberrations chromosomiques) ou pour évaluer la dose de radiation à travers des dommages chromosomiques (dosimétrie biologique);

- l'épidémiologie, à travers les populations concernées par des expositions environnementales, professionnelles ou médicales;

- les sciences humaines et sociales, c'est le cas du droit à la santé (évaluation des risques radioinduits ; prise en compte de la radiosensibilité individuelle; respect des règles de radioprotection et nouvelles recommandations, ...) mais aussi de la psychologie sociale qui étudie la perception des risques liés aux RI.

Enfin, notons que le terme « radiobiologie » est quelquefois utilisé par extension pour tous les effets liés aux ondes électromagnétiques ou aux UV. Cependant, les termes « électrobiologie » ou "photobiologie » devraient être préférés pour désigner ces disciplines spécifiques. Dans cet article, ne seront considérés que les effets des RI.

\section{Historique et évolutions de la radiobiologie française jusqu'à aujourd'hui}

\subsection{Période 1896-1918: Les français, pionniers reconnus en Europe et dans le monde}

La radiobiologie est née quelques mois après la découverte des rayons $\mathrm{X}$, dès que les pionniers des radiations ont pris conscience des conséquences cliniques d'une exposition aux radiations (Glasser, 1931). Il faut ici insister sur le fait que c'est justement en France et notamment à Lyon, grâce au mécénat des frères Lumière, que la radiobiologie a pris un essor particulier. En effet, quelques mois après la publication princeps de Roentgen, Etienne Destot répertorie déjà les différentes fractures possibles des membres inférieurs et supérieurs et Victor Despeignes réalise la première radiothérapie anti-cancéreuse (Foray, 2013; Foray et al., 2017). Entre 1906 et 1911, en réponse aux travaux des bordelais Bergonié et Tribondeau, Claudius Regaud propose les premières lois de la radiobiologie avant de prendre à Paris la direction de la Section de Biologie de l'Institut du Radium aux côtés de Marie Curie (Foray, 2012; Vogin et Foray, 2013).

\subsection{Période 1918-1954 : La prédominance de l'Institut Curie}

Après la première guerre mondiale, même si des travaux remarquables sont réalisés en province, l'Institut Curie dominera seul la radiobiologie française par l'action de
Claudius Regaud puis de son élève Antoine Lacassagne (Chamak, 2011). C'est à cette période que les 1000 premiers patients traités par radiothérapie furent pris en charge par Regaud et Coutard, nominés tous deux au Prix Nobel de Médecine. Ce fut le début de la radiothérapie fractionnée (Foray, 2012). La recherche française en radiobiologie atteint son apogée avec la création d'une chaire de radiobiologie expérimentale au Collège de France pour Antoine Lacassagne de 1941 à 1951. Cette chaire, unique dans l'histoire de l'enseignement, ne survivra pas à son fondateur (Chamak, 2011).

\subsection{Période 1954-1994: Redistribution des différents domaines de la radiobiologie}

Après le départ à la retraite d'Antoine Lacassagne en 1954, plusieurs événements géopolitiques et avancées technologiques furent à l'origine de 4 grands courants de la radiobiologie française : ceux-ci concernent le Commissariat à l'Énergie Atomique (CEA) et le Service de Santé des Armées (SSA), l'Institut Gustave-Roussy (IGR), l'Institut Curie et les laboratoires académiques (CNRS, INSERM, universités).

\subsubsection{La radiobiologie au CEA et au Service de Santé des Armées}

Les bombardements d'Hiroshima et de Nagasaki, l'étude de leurs conséquences et l'essor de l'énergie nucléaire civile ou militaire posèrent des questions sur les effets des faibles doses de radiation (mutagénèse et carcinogénèse radioinduites) mais aussi sur les fortes doses (radiopathologie) et la toxicité des radioéléments (radiotoxicologie). Impliquant la souveraineté nationale, ces thèmes furent d'abord naturellement traités par le CEA et le SSA. Notons que, progressivement, le besoin de mesure de la dose physique par analyse chromosomique (dosimétrie biologique) pérennisa les travaux en cytogénétique. En particulier, grâce aux travaux de l'Unité de Bernard Dutrillaux, le CEA devint leader dans cette technique. À Grenoble, le Laboratoire des Acides Nucléiques (LAN-CEA) de Jean Cadet traitait des dommages de l'ADN en général, et notamment des dommages de bases et de leur réparation.

Le problème des effets sanitaires des transuraniens et des produits de fission dispersés dans l'ensemble des hémisphères devint une source de préoccupation majeure. Au CEA, cet effort fut coordonné par Jacques Lafuma. En l'absence de données épidémiologiques suffisantes, la modélisation de ces effets fut abordée par la cancérogénèse expérimentale en relation étroite avec la communauté internationale rassemblée au sein de la Commission Internationale de Protection Radiologique(CIPR). Les possibilités d'extrapolation à l'homme furent évaluées à partir des données connues à la fois chez l'animal et chez l'homme (radium, expositions externes du corps entier) pour définir les facteurs de pondération nécessaires à une expression pertinente de la dose dans la perspective de la radioprotection.

En parallèle, la prise en charge des grands irradiés devint également une spécificité française d'excellence dans la suite de l'application aux irradiations accidentelles des greffes de moelle osseuses par le Pr Georges Mathé (1958) et le groupe du CEA dirigé par Henri Jammet. Les recherches autour du syndrome d'irradiation aiguë furent menées au Centre de Recherches du SSA (CRSSA) (Clamart puis Grenoble) et à 
l'origine de recherches précliniques étendues sur la radiosensibilité du système nerveux central (Louis Court), la thématique très militaire et "guerre froide» des lésions radiocombinées (Jean Drouet), les mécanismes de l'inflammation tissulaire radioinduite et la radiotoxicité hématologique (Francis Hérodin). Des études approfondies furent menées sur les agents radioprotecteurs chimiques (Marc Fatome) et les radiomitigateurs (Drouet et Herodin, 2014).

\subsubsection{La radiobiologie à l'Institut Gustave Roussy (IGR)}

Le développement des «bombes» au Cobalt, puis des accélérateurs pour la radiothérapie externe donnèrent un nouvel intérêt aux recherches en radiobiologie humaine pour les tumeurs et pour les tissus sains humains. C'est surtout sous l'impulsion du Pr. Maurice Tubiana que ce thème se développa à l'Institut Gustave-Roussy (IGR). Par la suite, EdmondPhilippe Malaise, directeur de recherche au CNRS fonda l'U247 INSERM, seule unité de recherche INSERM entièrement dédiée à la «radiobiologie des cellules normales et cancéreuses». Cette unité fut à l'origine de travaux fondateurs sur la radiosensibilité individuelle et des corrélations quantitatives entre les données radiobiologiques in vitro et les données cliniques in vivo (Foray et al., 2014). D'ailleurs, cette unité forma de nombreux radiothérapeutes qui sont aujourd'hui chefs de Département de Radiothérapie voire directeurs de Centres de lutte contre le cancer(CLCC). L'interaction avec les cliniciens devint l'une des spécificités de cette unité qui regroupa également les recherches sur l'effet oxygène (Marcelle Guichard) et les aspects cytogénétiques (Claude Parmentier et Martin Schlumberger) (Foray et al., 2014). Ainsi, tout en favorisant les synergies, furent traités dans la même unité les items majeurs de la radiobiologie, de la biochimie à la clinique avec une forte interaction avec les radiothérapeutes.

\subsubsection{La radiobiologie à Institut Curie}

La production industrielle des premiers radionucléides, le développement de la curiethérapie et les derniers travaux de Lacassagne sur les agents carcinogènes stimulèrent les recherches à l'Institut Curie sur les hydrocarbures cancérogènes et les psoralènes. Cette recherche se poursuivit avec Raymond Latarjet puis Emile Bisagni et Ethel Moustacchi, qui dirigea l'URA1292 CNRS «Anomalie dans l'expression des cytokines et radiosensibilité ». Même si ces recherches impliquèrent moins directement les RI, elles aboutirent à une meilleure connaissance fondamentale des mécanismes de réparation des dommages radioinduits de l'ADN. Les radiobiologistes de l'Institut Curie favorisèrent d'abord l'étude fondamentale de la réponse des micro-organismes (bactéries, levures) à différents stress. En parallèle, la caractérisation radiobiologique de l'anémie de Fanconi constitua l'un des premiers travaux sur une maladie génétique associée à une radiosensibilité (Papadopoulo et Moustacchi, 2005). À ce stade, on peut souligner l'interaction très forte des laboratoires de recherche de l'Institut Curie avec des généticiens cliniciens. Des travaux fondamentaux sur la radiosensibilité individuelle des patients furent initiés dans cette période par Ethel Moustacchi, Jean-Marc Cosset et Claire Alapetite. On note également au sein de l'U219 INSERM dirigée par Jean-Marc
Lhoste le début de travaux fondamentaux réalisés par l'équipe de Vincent Favaudon sur l'association concomitante des radiations et des drogues antitumorales cytotoxiques (néocarzinostatine, poisons des topoisomérases I et II), travaux qui se développeront par la suite au sein des Unités INSERM U350 et U612 en association avec de jeunes médecins inscrits au DEA de radiobiologie et qui mèneront à six thèses de doctorat.

\subsubsection{La radiobiologie dans les laboratoires universitaires}

Dans cette période, alors que les institutions citées plus haut se focalisaient sur les recherches en radiobiologie moléculaire, cellulaire et clinique, certains laboratoires académiques, principalement universitaires et/ou CNRS assurèrent des recherches d'excellence sur des aspects physico-chimiques fondamentaux qui n'étaient pas traités par les laboratoires décrits plus haut. Ce fut notamment le cas des recherches sur:

- les produits de la radiolyse de l'eau assurée par le Laboratoire de Chimie Physique, URA 400 CNRSUniversité Paris V dirigée par Christiane Ferradini, issue de l'Institut Curie (laboratoire d'Irène Joliot-Curie). Plus tard, ce furent Monique Gardès-Albert et Chantal Houée-Levin qui perpétuèrent ces approches (Gardes-Albert, 2006).

- les simulations de Monte-Carlo et les microdépôts d'énergie, principalement ceux déposés par les ions lourds (Groupe de Physique des Solides, URA 017 CNRS Université Paris 7, dirigé par Annie Chetioui) (Herve du Penhoat et al., 1999).

- les produits de la radiolyse de l'eau et les interactions DNA-protéines (notamment après irradiation par neutrons) sur ces complexes (URA 4301 CNRS-Centre de Biophysique Moléculaire, Orléans, dirigé Michel Charlier puis Mélanie Spotzheim-Maurizot) (Spotheim-Maurizot et Davidkova, 2011)

- la radiobiologie des radionucléides utilisés en médecine nucléaire (Département de Médecine Nucléaire du CHU Henri-Mondor de Créteil, dirigé par Pierre Galle) (Hindie et al., 2001).

\subsubsection{Synthèse de la période 1954-1994}

Le constat que l'on peut faire de cette période 1954-1994 est que tous les domaines de la radiobiologie étaient représentés en France, souvent à très haut niveau et toutes les institutions nationales de recherche étaient concernées. Une véritable interaction s'effectuait entre le monde académique représenté par une quinzaine de chercheurs INSERM/CNRS et les cliniciens, principalement des radiothérapeutes. De nombreuses autres spécialités médicales furent concernées par cette interaction. Cette cohésion et cette visibilité furent renforcées après l'accident de Tchernobyl (1986) par la création d'un DEA de radiobiologie où chaque institution était représentée et où chaque item était étudié en suivant un ordre chronologique cohérent (des événements radiophysiques aux événements cliniques radioinduits, des fortes aux faibles doses). Issus du DEA de radiobiologie (Paris V, Paris XI, Paris XII), de nombreux thésards, médecins ou scientifiques devinrent radiobiologistes en perpétuant dans chaque laboratoire d'accueil le savoir et le savoir-faire. 


\subsection{Période 1994-2004: Diminution drastique des effectifs et changements d'orientation pour certaines institutions}

\subsubsection{Situation à I'IGR}

La période 1994-2004 inclut la fin du mandat de l'U247 INSERM (IGR)-comme on l'a dit plus haut, seule unité de recherche académique dédiée entièrement à la radiobiologie. Les départs à la retraite de Edmond-Philippe Malaise, Marcelle Guichard et Claude Parmentier eurent également pour conséquence de mettre un arrêt dans les interactions entre les chercheurs radiobiologistes et les radiothérapeutes. C'est grâce à l'impulsion du Pr Jean Bourhis, chef du Département de Radiothérapie de l'IGR (création d'une Équipe d'Accueil 2710) puis de son successeur le Pr Eric Deutsch (création de l'Unité UMR 1030 «Radiothérapie moléculaire») que l'activité de recherche transversale reprendra avec les radiothérapeutes. Toutefois, le très faible renouvellement des postes académiques et l'absence de passage de relais entre chercheurs académiques et cliniciens ne purent pas compenser la perte de savoir et de savoirfaire : à partir de cette période, certains items fondamentaux de la radiobiologie ne furent plus traités en France.

\subsubsection{Situation à l'Institut Curie}

Sur le campus de Paris, on observa la même tendance avec le départ à la retraite d'Ethel Moustacchi. En revanche, sur le campus d'Orsay, l'Équipe de Vincent Favaudon (U350 INSERM) qui deviendra Unité 612 INSERM « Génotoxicologie, signalisation et radiothérapie expérimentale » et les Équipes de Dietrich Averbeck, Evelyne Sage et plus tard Marie Dutreix (UMR 2027 « Génotoxicologie et cycle cellulaire ») assurèrent le relais du savoir et du savoir-faire. Toutefois, une tendance apparut: celle de s'éloigner de la problématique clinique et humaine pour aborder des sujets mécanistiques très fondamentaux (ex : réparation et signalisation des dommages de l'ADN) avec des modèles de microorganismes (bactéries, levures) et de mammifères et des stress qui ne sont pas forcément radiatifs ou qui sortent des domaines de doses appliquées en clinique: les radiations ionisantes, "sujets d'étude » pour les radiobiologistes devinrent progressivement des «outils d'étude» pour les biologistes plus fondamentaux. Comme nous le verrons plus bas, la même tendance s'imposa au CEA.

\subsubsection{Situation dans les universités}

Le départ à la retraite de radiobiologistes universitaires, le non-renouvellement de leur poste et la fermeture définitive de certains laboratoires (ex: Orléans) sonnèrent le glas d'une radiobiologie française où tous les items de la discipline étaient représentés. Notons que la plupart des chercheurs atteints par la limite d'âge assuraient également les enseignements du DEA de radiobiologie (Paris XI, Paris XII, Paris V, Paris VI, IGR, Curie) (voir plus loin).

\subsubsection{Situation au CEA, à l'IRSN et à l'IRBA}

La même période fut marquée par la création de l'Institut de Radioprotection et de Sûreté Nucléaire (IRSN), issu de l'Office de protection contre les Rayonnements Ionisants (OPRI) et de l'Institut de Protection et de Sûreté Nucléaire (IPSN) puis du partage des ressources humaines avec le CEA.
Sans produire de nouveaux domaines de radiobiologie, cette création aboutit à une redistribution des sujets traités au sein de ces deux institutions (IRSN et CEA). En particulier, l'IRSN traita notamment sous la direction de Patrick Gourmelon de la dosimétrie physique (Alain Rannou, Jean-François BottellierDepois) et biologique (Philippe Voisin, Laurence Roy) et des processus de l'inflammation tissulaire (Jean-René Jourdain, Marc Benderitter).

Le CEA maintint son action en radiotoxicologie et continua à soutenir des recherches en cytogénétique (Laure Sabatier), ainsi que sur la réparation des dommages de l'ADN induits par les espèces radicalaires réactives de l'oxygène (Serge Boiteux). De plus, le CEA aborda la réponse aux radiations des kératinocytes (Michèle Martin) et des cellules souches (Paul-Henri Roméo) et les signatures des cancers radioinduits (Sylvie Chevillard). En plus de ces items qui concernent les cellules humaines, et comme à l'Institut Curie, des recherches s'effectuèrent sur des sujets bien plus fondamentaux comme la réparation de l'ADN, les arrêts du cycle cellulaire et la mort cellulaire avec des modèles non humains (microorganismes, rongeurs).

Les recherches du CRSSA (devenu Institut de Recherches Biomédicales des Armées (IRBA) en 2009 et transféré à Brétigny-sur-Orge en 2013) se concentrèrent largement sur la compréhension et la prévention du syndrome d'irradiation aigüe et le développement de contre-mesures de radiomitigation. Un effort conséquent fut initié pour accroître la transversalité et intégrer les nouvelles approches de thérapie cellulaire dans le savoir-faire aboutissant à la reconnaissance, la décennie suivante, de l'îlot Percy comme centre d'excellence pour le traitement des irradiés. Pour autant, la période tirant bénéfice de la fin de la guerre froide, ne fut pas favorable au développement de la discipline.

\subsubsection{Synthèse de la période 1994-2004}

La période 1994-2004 fut tout d'abord marquée par le départ à la retraite de nombreux chercheurs académiques (CNRS et INSERM), postes qui ne furent pas renouvelés alors qu'ils représentaient des thématiques fondamentales de la radiobiologie (notamment les aspects physico-chimiques. En parallèle, la loi LMD (Licence Maîtrise Doctorat) de 2004 contribua à fondre les hyper-disciplines dans des systèmes d'enseignement plus généraux : les DEA disparurent progressivement au profit des masters. Privé de certains de ses enseignants, le DEA de radiobiologie ne fit pas exception et un enseignement de radiobiologie fut transformé en une option ou parcours du Master de cancérologie de l'Université Paris XI (voir plus loin). Le DEA de radiobiologie était devenu quasiment l'unique source de « production » de radiobiologistes en France, indifféremment du statut de médecin ou de scientifique et offrait une très grande visibilité aux étudiants comme aux décideurs. Avec sa disparition, tout un pan du savoir et savoir-faire de radiobiologie allait disparaître dans le domaine de la cancérologie.

\subsection{Période actuelle (2004-2021): de nombreuses thèses, peu de recrutements de radiobiologistes académiques, une demande forte des cliniciens}

\subsubsection{Qu'appelle-t-on radiobiologiste aujourd'hui?}

Depuis une dizaine d'années, de nombreux chercheurs biologistes, microbiologistes utilisent les RI et d'autres agents 
cassants comme des modèles de stress génotoxique pour vérifier la validité de leurs hypothèses mécanistiques (c'est l'approche « radiations-outils »). Sont-ils pour autant tous radiobiologistes et experts dans les effets biologiques et cliniques des radiations ionisantes? Répondre à cette question est essentiel puisqu'au dernier recensement effectué à ce jour (collectif, 2011), 96 équipes ont répondu, représentant 427,2 équivalent temps plein recherche (ETPR). Ces chiffres du rapport 2011 de AVIESAN peuvent interroger puisqu'ils dépassent les forces vives en radiobiologie des USA. L'explication est simple et est aussi représentative du passage de l'approche «radiations-sujet» à l'approche «radiations-outils ». Il faut en effet rappeler que le questionnaire préparé par AVIESAN sur 2010 et 2011 a été envoyé à toutes les structures de recherche en France. Ce sondage démontre l'évidence d'un usage massif des RI dans les recherches en génotoxicologie en France mais ne distingue pas spécifiquement les experts en radiobiologie, qui font des RI leur sujet d'étude. D'ailleurs, le rapport du CEA publié en 1994 (CEA, 1994) (soit 16 ans avant le rapport AVIESAN), mentionnait 42 Équipes et environ 50 ETPR (CEA, 1994). Mais cette fois, cette évaluation fut effectuée sur un recensement adressé directement par les auteurs du rapport aux laboratoires qu'ils avaient considérés comme étant des «laboratoires de radiobiologie ». Le constat de cette analyse est que nous ne disposons pas à l'heure actuelle d'une liste précise d'experts en radiobiologie en France.

Afin de recenser de façon objective, les forces vives de la radiobiologie française, il faut donc d'abord (re-)définir ce qu'est un radiobiologiste et une Équipe de recherche en radiobiologie :

- aujourd'hui, on peut raisonnablement définir un radiobiologiste comme un chercheur ou un clinicien ayant soutenu une thèse de sciences en radiobiologie ou, pour les générations antérieures ayant passé sa thèse avant les années 1990, un chercheur ou clinicien ayant travaillé au moins 10 ans sur un projet de recherche où les effets des RI constituent le sujet principal. Ce «temps zéro » des années 1990 correspond à la création du DEA de radiobiologie.

- d'après les dernières recommandations de l'INSERM, une Équipe de recherche est une entité opérationnelle élémentaire d'une Unité de recherche. L'Équipe se définit par ses objectifs scientifiques et par les moyens (financiers, en équipement et en personnel) dont elle dispose pour réaliser un programme de recherche propre. L'équipe doit comporter au moins un chercheur titulaire temps plein appartenant à un EPST ou un EPIC et la taille raisonnable d'une équipe pourra être d'au moins de 5 ETPR incluant les post-doctorants.

- une Équipe de recherche en radiobiologie peut donc se définir comme une Équipe comportant au moins un radiobiologiste et réalisant au moins un projet de recherche en radiobiologie nécessitant l'utilisation régulière de RI et visant à mesurer leurs effets à travers ses conséquences physiques, chimiques, moléculaires, cellulaires, tissulaires ou macroscopiques telles que définis plus haut.

\subsubsection{Combien y-a-t-il de docteurs en radiobiologie en France?}

Tout comme les définitions ci-dessus, il est également difficile de définir et de répertorier les thèses en radiobiologie avec les moyens actuels. En effet, le site www.theses.fr qui pourtant rassemble toutes les thèses soutenues en France ne possède pas de moteur de recherche suffisamment précis car, selon les saisies informatiques et les règles en vigueur dans les Universités, le terme «radiobiologie» peut apparaitre indifféremment dans les intitulés ou les spécialités des Ecoles Doctorales voire dans l'intitulé du laboratoire des membres du Jury. Ainsi, en tentant de répertorier les thèses de radiobiologie à la lecture de leur titre et de leur résumé pour vérifier leur contenu, on peut raisonnablement considérer que, depuis les années 1990, entre 136 et 184 (soit une moyenne de 160) thèses de sciences en radiobiologie (comme défini plus haut) ont été soutenues en France (Fig. 1).

L'analyse de l'évolution du nombre de thèses soutenues par an montre 3 phases :

- de 1990 à 1997 environ, une augmentation régulière d'environ 1 thèse supplémentaire chaque année. Ces thèses initiées 3 à 4 ans auparavant correspondent à la production régulière du DEA de radiobiologie créé à la suite de l'accident de Tchernobyl (1986);

- de 1998 à 2010 environ, une baisse puis une stagnation (environ 6 thèses soutenues par an) probablement dues au départ à la retraite des chercheurs académiques et à la fermeture de certains laboratoires d'accueil du DEA de radiobiologie ;

- de 2010 à 2020, une augmentation lente mais régulière d'environ 1 à 2 thèses supplémentaires par an. Ainsi, comme beaucoup d'autres disciplines, le nombre de thèses en radiobiologie augmente régulièrement depuis 1990, c'est une tendance générale universitaire. Cependant, il faut évoquer 2 événements importants qui ont pu contribuer spécifiquement à cette nouvelle tendance: l'accident de Fukushima, qui a pu stimuler l'attente sociétale et le séminaire AVIESAN de Montpellier de 2010 qui, sur l'initiative des 3 ITMOs Cancer, Hématologie et Technologie pour la Santé, en invitant tous les laboratoires nationaux impliqués dans la recherche en radiobiologie a tenté de redynamiser la radiobiologie française et d'identifier les causes de son déclin (collectif, 2011). Notons enfin que cette période correspond également à la création de la Société Internationale de Radiobiologie de Langue Française (SIRLaF) (aujourd'hui Société Française de Biologie des Radiations (SFBR)), qui a pu favoriser, par ses congrès bisannuels les interactions entre chercheurs.

Même si les thèses soutenues en radiobiologie sont en augmentation régulière depuis 2010 , elles n'aboutissent pas toutes, loin de là, à des postes académiques de radiobiologistes. De plus, cette évolution n'indique pas clairement si certains pans de la radiobiologie sont toujours étudiés et représentés. Allons plus loin dans l'analyse.

\subsubsection{Le devenir des docteurs en radiobiologie en France}

Pour analyser le devenir des doctorants-radiobiologistes, nous avons fait des recherches systématiques à la fois sur Internet et dans les archives universitaires et des différentes institutions. Malheureusement, le parcours post-doctoral de tous les doctorants-radiobiologistes qui sont répertoriés dans la figure 1 n'a pu être identifié. C'est donc sur un panel de 145 doctorants-radiobiologistes sur 160 environ (soit 90,6\%) issus du DEA de radiobiologie ou du Master de cancérologie option radiobiologie que nous avons pu travailler. 


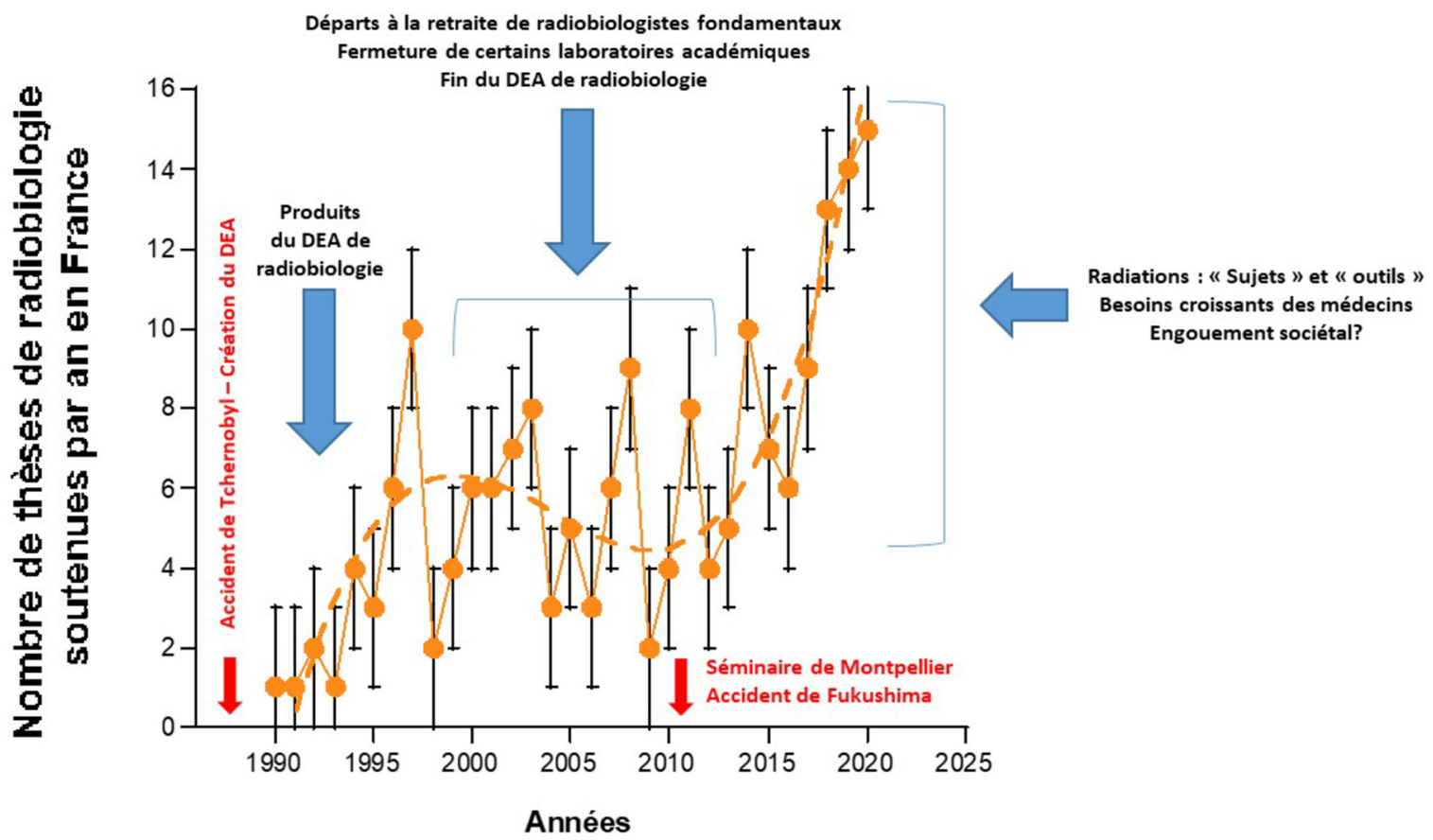

Fig. 1. Nombre de thèses en radiobiologie soutenues en France. Les données sont issues du site thèses.fr en utilisant « radiobiologie » pour motclé mais en ré-analysant le titre, le résumé et la composition du jury. Ont été omises de cette sélection, les quelques thèses dont le sujet n'est pas directement lié aux radiations et dont le mot-clé est lié seulement à l'intitulé du laboratoire d'un des membres du jury. Une barre d'erreur correspondant à \pm 2 thèses, a été systématiquement appliquée.

La période de référence va de 1990 à 2017 (Fig. 2). On observe que:

- les médecins représentaient plus de $45 \%$ des doctorants en radiobiologie entre 1990 et 1994 (dont 36,5\% pour les seuls radiothérapeutes), ce qui soulignait bien l'attrait de la radiobiologie pour les radiothérapeutes. Cette proportion tombe à $4 \%$ entre 2014 et 2017.

- pour les EPST (INSERM et CNRS), un nombre négligeable (si ce n'est nul) de docteurs en radiobiologie ont été recrutés au CNRS ou à l'INSERM depuis 2005 en tant que chercheurs

- pour les EPIC (CEA et IRSN), il n'y a plus eu de docteurs en radiobiologie recrutés au CEA depuis 2010. Par contre, les recrutements à l'IRSN restent faibles mais jamais nuls (18\% des doctorants entre 1990 et 1994). Leur nombre a cependant été divisé par 4 en presque 25 ans ( $4 \%$ entre 2014 et 2017).

- entre 2014 et 2017, au moins $13 \%$ des docteurs en radiobiologie rejoignent le secteur privé, au moins $13 \%$ acquièrent des postes à l'étranger et au moins $13 \%$ également deviennent post-doctorants à l'étranger, c'est-à-dire qu'au moins $40 \%$ environ sortent du système académique. Les postdoctorants en France représentent une minorité $(4 \%)$ et le recul n'est pas encore suffisant pour considérer leur devenir mais peu ont postulé aux concours INSERM ou CNRS.

\subsubsection{Paradoxe entre le nombre de thèses et le nombre de recrutements académiques}

Aujourd'hui, le système universitaire produit chaque année en France environ une dizaine de docteurs en radiobiologie.
Peut-être n'est-ce qu'un effet Fukushima. En effet, ces thèses ne sont plus forcément produites par les laboratoires académiques de radiobiologie puisque ceux-ci ont quasiment disparu. En fait, ces données suggèrent que l'usage des radiations a pu changer de nature: on passe alors des radiations « sujets » aux radiations « outils ». Cette transition a-t-elle une conséquence sur la nature et la qualité des publications scientifiques publiées par les laboratoires français? Nous y revenons plus loin.

Le recrutement de radiobiologistes au niveau académique (CNRS, INSERM, CEA) en baisse constante est désormais nul : nous ne recrutons plus les thésards qui sont produits par le système universitaire. Ceux-ci disparaissent des cursus ou la seule issue possible aujourd'hui reste l'embauche dans le secteur privé ou à l'étranger, postes qui ne correspondent pas forcément à un besoin en radiobiologie. Ces radiobiologistes formés en France ne constituent plus le vivier qui permettrait de renouveler les effectifs des laboratoires de radiobiologie, même à leur retour de l'étranger ou du privé.

\subsubsection{Les radiations ionisantes: outil ou sujet?}

Comme on l'a dit plus haut, certaines institutions et laboratoires ont fait le choix de sujets de recherche plus fondamentaux réalisés avec des modèles cliniques non humains comme notamment les microorganismes (bactéries, levures). Ces études ont pu éventuellement s'étendre à des modèles de mammifères. Il faut tout d'abord noter qu'une telle tendance est générale et non spécifique à la France. Cette tendance, facilement vérifiable dans les index des revues internationales, répond d'abord à une demande forte des tutelles à augmenter le facteur d'impact des publications des 

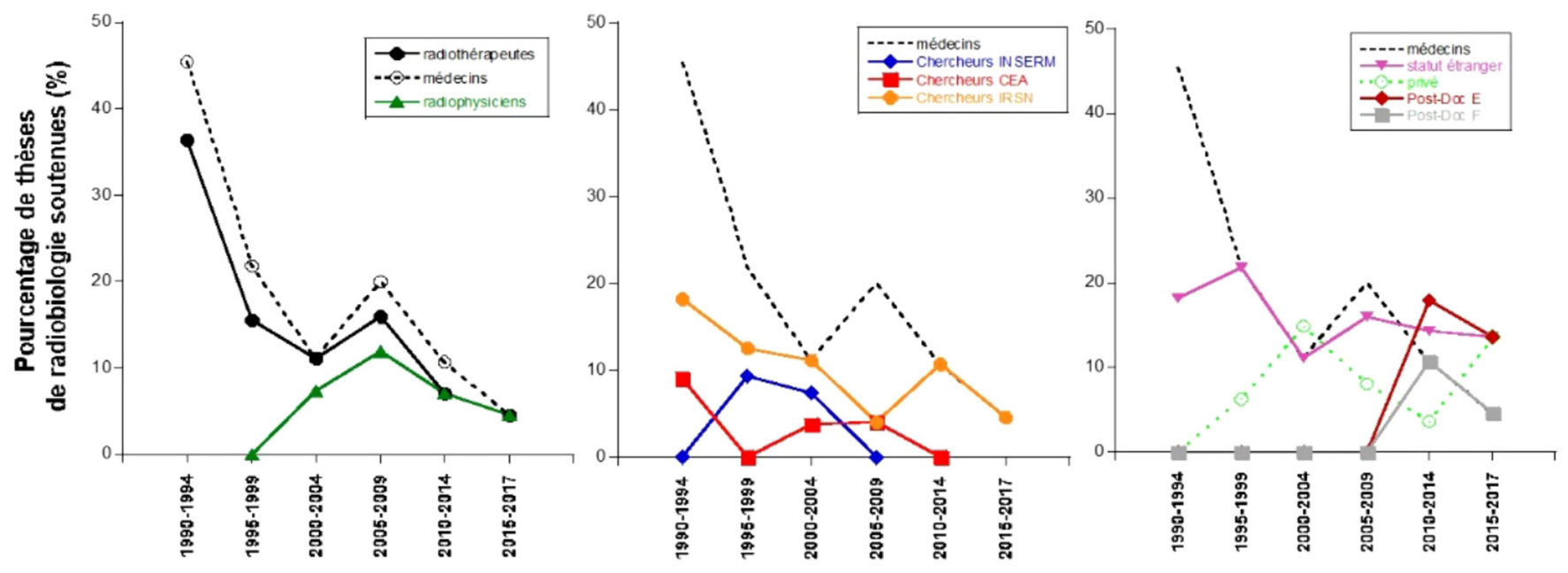

Fig. 2. Devenir de 145 sur 160 doctorants-radiobiologistes issus du DEA de radiobiologie ou du Master de Cancérologie option radiobiologie entre 1990 et 2017. Le devenir des doctorants a été exprimé en pourcentage sur les périodes de référence indiquées (E désigne «à l'étranger» et $\mathrm{F}$ désigne «en France»).

laboratoires qu'elles soutiennent: il est indubitable que des travaux mécanistiques très fondamentaux trouvent mieux leur place dans des journaux à haut facteur d'impact que des travaux applicatifs à la radiothérapie, la radiologie ou à la mesure du risque: les travaux de radiobiologie humaine, sont généralement publiés dans des journaux de spécialité, moins généralistes et donc de facteur d'impact plus bas. Notons par exemple que les premiers journaux de radiothérapie ou de radiologie ne dépassent pas un facteur d'impact de 7. Ceux des journaux de radiobiologie sont inférieurs à 3. Ainsi, la fondamentalisation des sujets de radiobiologie traités par certains laboratoires du CEA, de l'Institut Curie et, à moindre échelle de l'IGR a eu pour effet d'augmenter le facteur d'impact des publications en produisant une recherche d'excellence. En soi, une telle recherche pouvait se substituer à ce que la recherche académique (notamment CNRS) aurait pu produire. Cette réorientation a eu cependant pour effet de s'éloigner des thématiques proches de la clinique, utiles pour les radiothérapeutes ou les radiologues. En effet, bien que les travaux sur les microorganismes (qui sont généralement effectués avec des centaines voire des milliers de Gy) ont fait progresser les connaissances fondamentales sur le cycle cellulaire ou certaines voies de signalisation, les données ne peuvent être extrapolées facilement ni à la radiothérapie (doses de l'ordre du Gy), ni aux faibles doses inférieures au mGy, ni à la cancérogénèse, ni à la réglementation sur la radioprotection.

En même temps que le choix de modèles cellulaires plus éloignés de la clinique, se rajoutait l'usage d'agents chimiques et de conditions expérimentales (notamment en termes de doses ou de concentration) encore moins compatibles avec des conditions réalistes applicables à l'homme. Il n'est pas rare de voir des publications de biologie moléculaire effectuées avec des doses de plusieurs dizaines de Gy en dose unique alors que la dose létale immédiate pour l'homme est d'environ $12 \mathrm{~Gy}$, que la dose létale moyenne (DL50) admise se situe autour de 4,5 Gy, que la dose moyenne par session en radiothérapie est de 2 Gy et que le seuil consensuel des cancers solides radioinduits est de $200 \mathrm{mGy}$. On perçoit bien ici toute la différence entre des recherches très fondamentales qui perdent à la fois leur lien avec la clinique et requièrent une utilisation plutôt épisodique des RI (au gré des besoins) d'une part, et des recherches plutôt transversales et appliquées, proches des conditions cliniques et qui impliquent un usage quotidien des RI, d'autre part. Comme on l'a déjà suggéré plus haut, on peut qualifier la première tendance d'une recherche où les radiations constituent un «outil» non permanent. La seconde est la voie « historique » et classique des RI en tant que «sujet de recherche». Évidemment, ces différents choix ne conditionnent pas la valeur et la qualité des recherches mais elles ne requièrent pas la même expertise ni le même savoir-faire : c'est pourtant la seconde voie, qui produit les experts en radiobiologie et radiopathologie qui répondent aux journalistes au moment des crises.

De plus, l'approche historique des «radiations-sujet» nécessite inévitablement pour le laboratoire l'accès à des irradiateurs ou des sources de RI, notamment médicaux. C'est pourquoi le site idéal pour les laboratoires de radiobiologie se situe aujourd'hui près des services de radiothérapie et notamment des CLCC. Rappelons d'ailleurs que les moyens d'irradiation des radiobiologistes experts des aspects physicochimiques de l'irradiation restaient plutôt expérimentaux et éloignés des centres médicaux (ex: Orléans, Grenoble). Les CLCC français apparaissent ainsi comme des sites propices pour l'installation de laboratoires qui auraient choisi les radiations-sujets. On observe d'ailleurs une volonté croissante des CLCC ou de centres proches des CLCC à développer de nouveaux laboratoires comme à Lille, Nice, Rennes, ... s'ajoutant à ceux déjà établis à Caen, Paris, Villejuif, Lyon, Montpellier, Nantes, Strasbourg et Toulouse. Cette démarche a l'avantage de regrouper sur un même site, radiothérapeutes, radiophysiciens et radiobiologistes.

À l'inverse, le choix de l'approche «radiations-outils» peut avoir une conséquence sur la lisibilité du laboratoire mais aussi sur le choix des revues scientifiques où les travaux sont publiés. Afin d'illustrer plus concrètement les conséquences d'une telle réorientation thématique, nous avons recensé les publications produites par des groupes de recherche français en tant qu'investigateurs principaux dans les revues principales 

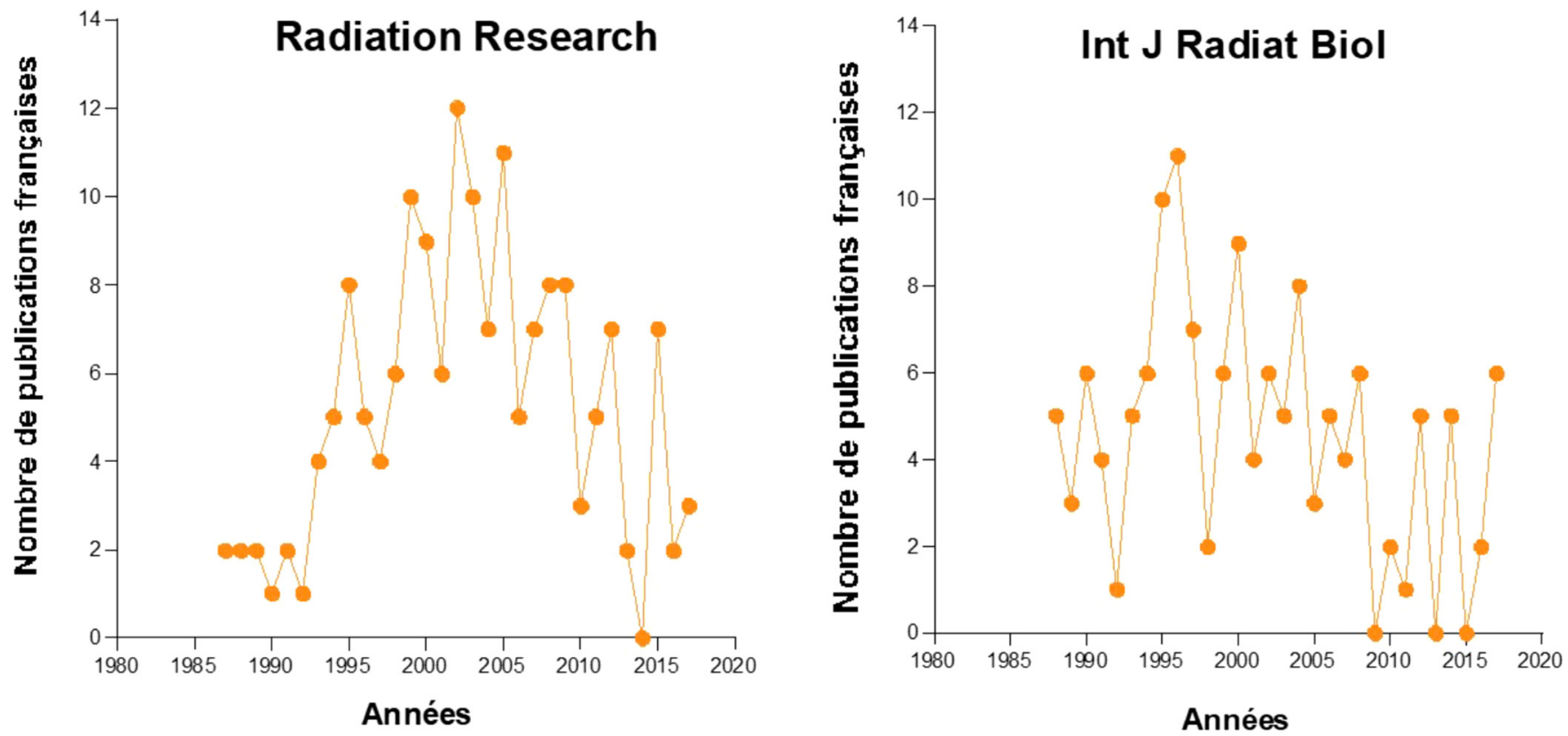

Fig. 3. Nombre de publications françaises dans les journaux Radiation Research ou International Journal of Radiation Biology entre 1990 et 2017. Nous n'avons considéré que les publications dans lesquelles au moins le premier ou bien le dernier auteur dépend d'un laboratoire français.

de radiobiologie, «Radiation Research» et «International Journal of Radiation Biology» (Fig. 3). La figure 3 montre qu'entre 1986 et les années 2000 , le nombre d'articles publiés dans ces deux revues internationales par les groupes de recherche français augmente jusqu'à au moins 10 publications par an. À l'inverse, après les années 2000, ce nombre baisse régulièrement. Il faut cependant noter qu'une analyse fine de ces données montre clairement que le nombre de publications produites par l'IRSN et l'IRBA dans ces journaux reste constant alors que le nombre de publications publiées par les autres institutions françaises (INSERM, CNRS, CEA) décroît significativement. Ces données illustrent bien à la fois la tendance de certains groupes à publier dans d'autres journaux plus généralistes et la disparition progressive des groupes académiques qui entraîne la baisse de la production scientifique française en matière de radiobiologie «radiations-sujet ».

\subsubsection{Master ou DU de radiobiologie?}

On observe qu'il y a de moins en moins de médecins et notamment de radiothérapeutes qui suivent le Master de cancérologie option radiobiologie (qui s'est substitué au DEA de radiobiologie de Paris XI) et qui effectuent une thèse de doctorat en radiobiologie.

Il n'est d'ailleurs pas besoin de faire beaucoup de statistiques tant cette tendance est évidente. Le DU de radiobiologie créé à Lyon par Claire Rodriguez-Lafrasse n'a pas corrigé cette tendance. En effet, dans le cursus d'un spécialiste clinicien, effectuer une thèse est un investissement considérable en temps et un choix difficile d'organisation. Effectuer un master impose de réaliser un stage dans un laboratoire de recherche en produisant un mémoire résumant des travaux de laboratoire qui peuvent être parfois éloignés de la réalité clinique. C'est pourquoi, un DU de radiobiologie qualifiant qui propose la même qualité d'enseignement (souvent réalisé par les mêmes personnes que le master) mais qui ne nécessite pas de stage en laboratoire, est apparu comme un bon compromis pour les médecins. Il faut toutefois insister sur le fait que master et DU n'ont évidemment pas la même finalité ni n'attribuent une expertise égale.

La création du DU de radiobiologie de Paris XI et l'IGR en 2006 a coïncidé ainsi avec une baisse des effectifs dans le Master de cancérologie option radiobiologie. En 2015, les effectifs des médecins ne dépassaient pas 10 au master alors qu'ils atteignaient déjà la trentaine au DU de radiobiologie. Evidemment, le DU de radiobiologie n'est pas une option adaptée pour les scientifiques désirant effectuer une thèse. Ceux-ci doivent alors se diriger de plus en plus vers des masters où la radiobiologie n'est pas enseignée. Leur formation dans cette discipline s'effectuera alors «sur le tas » dans les laboratoires de radiobiologie, ce qui aura pour conséquence, une fois encore, une baisse de savoir, de savoirfaire, d'expertise et l'augmentation d'une certaine précarité.

\section{Conclusions et perspectives}

À travers cette revue, nous avons vu que notre pays est historiquement attaché à la radiobiologie et de grandes figures de cette hyper-discipline sont françaises. L'accident de Tchernobyl avait contribué à la création d'un DEA de radiobiologie où toutes les compétences et les institutions étaient réunies de façon complémentaire. Cette cohésion et cette cohérence assuraient un savoir, un savoir-faire et un haut niveau d'expertise tant pour les médecins que les scientifiques. Le résultat a été une remarquable émulation et une production scientifique de grande qualité par rapport aux autres pays développés.

Malheureusement, la dilution de l'enseignement de la radiobiologie dans la cancérologie, le non-renouvellement des 
postes académiques concernant la radiobiologie et la fermeture de certains laboratoires où des aspects fondamentaux de la réponse aux RI étaient étudiés ont initié le déclin de la radiobiologie française des années 1998 à 2010.

De plus, de nouvelles tendances (souvent mondiales) comme le changement d'orientations stratégiques vers des publications dans des journaux à plus haut facteur d'impact, l'utilisation des RI comme outils et non comme sujets d'étude et une offre d'enseignement universitaire différente et spécifique pour les médecins ont contribué à laisser des pans entiers de la discipline sans soutien ni remplacement des postes académiques vacants (aspects physico-chimiques, biophysiques, radiopathologie, immunitaires, ...).

L'accident de Fukushima n'a pas produit les mêmes effets que celui de Tchernobyl, à l'exception d'un certain intérêt pour les effets des RI dans le grand public, rendant plus vives encore, l'attente sociétale et certaines interrogations. En l'absence d'experts et de débats contradictoires, les plages médiatiques sont ainsi laissées aux adeptes de théories farfelues, de désinformations, de manipulations voire de thèses complotistes : c'est déjà le cas pour les retombées des accidents de Tchernobyl et de Fukushima.

Cette perte de savoir et de savoir-faire n'est pas spécifiquement française. En effet, en Europe, notamment dans le rapport 2012 de l'Agence de l'Énergie Nucléaire dépendant de l'OCDE (OECD, 2012) comme aux USA (Toohey, 2017), de nombreux chercheurs s'accordent à déplorer cette situation. Toutefois, dans le pays le plus nucléarisé du monde, cette perte de savoir et de savoir-faire pour des pans entiers de la discipline est encore plus visible. Il importe donc d'assurer de façon optimale la formation et le recrutement académique des experts de demain qui devront, dans le cadre de leurs activités de recherche, non seulement répondre aux défis scientifiques mais aussi, comme la situation actuelle liée au Covid le suggère, maîtriser la communication sur le risque.

\section{Références}

ASN. 2019. Arrêté du 8 février 2019 portant homologation de la décision n ${ }^{\circ} 2019-D C-0660$ de l'Autorité de sûreté nucléaire du 15 janvier 2019 fixant les obligations d'assurance de la qualité en imagerie médicale mettant en œuvre des rayonnements ionisants. JORF.

Bourguignon M. 2020. Plaidoyer pour un soutien fort et durable à la recherche en radiobiologie pour optimiser la radioprotection. Radioprotection 55: 159-62.

Britel M, Foray N, Preau M. 2015. Personalized medicine in radiotherapy: Practitioners' perception. Santé Publique 27: 669-77.

Britel M, Bourguignon M, Foray N. 2018. Radiosensitivity: A term with various meanings at the origin of numerous confusions. A semantic analysis. Int. J. Radiat. Biol. 94: 503-12.

Cannon G, Kiang JG. 2020. A review of the impact on the ecosystem after ionizing irradiation: Wildlife population. Int. J. Radiat. Biol.: $1-9$.

Cavallero S, Riccobono D, Drouet M, Francois S. 2020. MSC-derived extracellular vesicles: new emergency treatment to limit the development of radiation-induced hematopoietic syndrome? Health Phys. 119: 21-36.
CEA. 1994. Radiobiologie: besoins, moyens et projets dans la France de 1994. Paris, France: Commissariat à l'Énergie Atomique.

Chamak B. 2011. Cent ans de recherches en cancérologie. Le rôle d'Antoine Lacassagne (1884-1971). Paris : Éditions Glyphe.

collectif A-r. 2011. Recherches françaises en radiobiologie. Montpellier, France: AVIESAN.

Cosset JM, Mornex F, Eschwege F. 2013. Hypofractionation and radiotherapy: The eternal return. Cancer Radiother. 17: 355-62.

Ding D, Zhang Z, Lei Z, Yang Y, Cai T. 2016. Remediation of radiocesium-contaminated liquid waste, soil, and ash: A mini review since the Fukushima Daiichi Nuclear Power Plant accident. Environ. Sci. Pollut. Res. Int. 23: 2249-63.

Drouet M, Herodin F. 2014. Mitigating radiation-induced toxicity: An overview of new approaches developed at the French Military Biomedical Research Institute. Health Phys. 106: 682-8.

Drouet M, Ménager M. 2019. Quelles réponses aux menaces chimiques, biologiques et radiobiologiques. L'actualité chimique 440: $1-2$.

Endesfelder D, Oestreicher U, Kulka U, Ainsbury EA, Moquet J, Barnard S, Gregoire E, Martinez JS, Trompier F, Ristic Y, Woda C, Waldner L, Beinke C, Vral A, Barquinero JF, Hernandez A, Sommer S, Lumniczky K, Hargitai R, Montoro A, Milic M, Monteiro Gil O, Valente M, Bobyk L, Sevriukova O, Sabatier L, Prieto MJ, Moreno Domene M, Testa A, Patrono C, Terzoudi G, Triantopoulou S, Histova R, Wojcik A. 2021. RENEB/EURADOS field exercise 2019: Robust dose estimation under outdoor conditions based on the dicentric chromosome assay. Int. J. Radiat. Biol. 97: 1181-98.

Foray N. 2012. Claudius Regaud (1870-1940): A pioneer of radiobiology and radiotherapy. Cancer Radiother. 16: 315-21.

Foray N. 2013. Victor Despeignes (1866-1937): How a hygienist became the first radiation oncologist. Cancer Radiother. 17: 244-54.

Foray N, Badie C, Alsbeih G, Lambin P, Geara F, Taghian AG, Deschavanne P, Gueulette J, Courdi A, Chavaudra N, Fertil B. 2014. Edmond-Philippe Malaise (1930-2013): A lifetime of perseverance leads to the cellular definition of intrinsic radiosensitivity. Int. J. Radiat. Oncol. Biol. Phys. 88: 1215-7.

Foray N, Bourguignon M, Hamada N. 2016. Individual response to ionizing radiation. Mutat. Res. Rev. 770: 369-86.

Foray N, Amiel M, Mornex R. 2017. Etienne Destot (1864-1918) ou l'autre père de la radiologie française. Cancer Radiother. 21: 138-47.

Friedl AA, Prise KM, Butterworth KT, Montay-Gruel P, Favaudon V. 2021. Radiobiology of the flash effect. Med. Phys.

Gagna G, Pegorie A, Entine F, Michel X, Dondey M, Amabile JC. 2016. Missions of the French Defense Radiation Protection Service Concerning The Medical Management of Radiocontaminated Patients. Health Phys. 111: 223-6.

Gardes-Albert M. 2006. Physico-chemical aspects of reactive oxygen species. Ann. Pharm. Fr. 64: 365-72.

Glasser O. 1931. Wilhelm Conrad Röntgen and the early history of roentgen rays. Berlin: Julius Springer.

Herve du Penhoat MA, Fayard B, Abel F, Touati A, Gobert F, Despiney-Bailly I, Ricoul M, Sabatier L, Stevens DL, Hill MA, Goodhead DT, Chetioui A. 1999. Lethal effect of carbon K-shell photoionizations in Chinese hamster V79 cell nuclei: Experimental method and theoretical analysis. Radiat. Res. 151: 649-58.

Hindie E, Petiet A, Bourahla K, Colas-Linhart N, Slodzian G, Dennebouy R, Galle P. 2001. Microscopic distribution of iodine radioisotopes in the thyroid of the iodine deficient new-born rat: Insight concerning the Chernobyl accident, Cell. Mol. Biol. (Noisy-le-Grand) 47: 403-10. 
Joubert A, Vogin G, Devic C, Granzotto A, Viau M, Maalouf M, Thomas C, Colin C, Foray N. 2011. Radiation biology: Major advances and perspectives for radiotherapy. Cancer Radiother. 15: 348-54.

Lindberg JCH. 2021. J'accuse.!: The continuous failure to address radiophobia and placing radiation in perspective. J. Radiol. Prot. 41.

Mazal A, Prezado Y, Ares C, de Marzi L, Patriarca A, Miralbell R, Favaudon V. 2020. FLASH and minibeams in radiation therapy: The effect of microstructures on time and space and their potential application to protontherapy. Br. J. Radiol. 93: 20190807.

Mazeron JJ, Maugis A, Barret C, Mornex F. 2005. Techniques d'irradiation des cancers. La radiothérapie conformationnelle. Paris, France: Maloine.

Médecine ANd. 2015. De l'usage des rayons $X$ en radiologie (diagnostique et interventionnelle), à l'exclusion de la radiothérapie. Rapport et recommandations. Paris, France: Académie National de Médecine.
OECD N-. 2012. Nuclear education and training: From concern to capability. Paris, France: Organisation for Economic Co-operation and Development (OECD).

Papadopoulo D, Moustacchi E. 2005. Fanconi anemia: Genes and function(s) revisited. Med. Sci. (Paris) 21: 730-6.

Restier-Verlet J, El-Nachef L, Ferlazzo ML, Al-Choboq J, Granzotto A, Bouchet A, Foray N. 2021. Radiation on earth or in space: What does it change? Int. J. Mol. Sci. 22: 3739.

Riccobono D, Valente M, Drouet M, Calamai F, Abriat A. 2018. French policies for victim management during mass radiological accident/attacks. Health Phys. 115: 179-84.

Spotheim-Maurizot M, Davidkova M. 2011. Radiation damage to DNA in DNA-protein complexes. Mutat. Res. 711: 41-8.

Toohey RE. 2017. Thirteenth Annual Warren K. Sinclair Keynote Address: Where Are the Radiation Professionals (WARP)? Health Phys. 112: 121-25.

Vogin G, Foray N. 2013. The law of Bergonie and Tribondeau: A nice formula for a first approximation. Int. J. Radiat. Biol. 89: 2-8.

Citation de l'article: Foray N, Averbeck D, Cosset J-M, Drouet M, Favaudon V, Masse R. 2021. La radiobiologie française : historique, constats et enjeux. Radioprotection 56(4): 263-273 